\title{
Storyboard-Based Empirical Modeling of Touch Interface Performance
}

\begin{tabular}{|c|c|}
\hline Alix Goguey & Géry Casiez \\
\hline University of Saskatchewan & Université de Lille \\
\hline Canada & Inria \\
\hline alix.goguey@usask.ca & $\begin{array}{l}\text { France } \\
\text { gery.casiez@univ-lille.fr }\end{array}$ \\
\hline Andy Cockburn & Carl Gutwin \\
\hline University of Canterbury & University of Saskatchewan \\
\hline $\begin{array}{l}\text { New Zealand } \\
\text { andy@cosc.canterbury.ac.nz }\end{array}$ & $\begin{array}{l}\text { Canada } \\
\text { gutwin@cs.usask.ca }\end{array}$ \\
\hline
\end{tabular}

\begin{abstract}
Touch interactions are now ubiquitous, but few tools are available to help designers quickly prototype touch interfaces and predict their performance. For rapid prototyping, most applications only support visual design. For predictive modelling, tools such as CogTool generate performance predictions but do not represent touch actions natively and do not allow exploration of different usage contexts. To combine the benefits of rapid visual design tools with underlying predictive models, we developed the Storyboard Empirical Modelling tool (StEM) for exploring and predicting user performance with touch interfaces. StEM provides performance models for mainstream touch actions, based on a large corpus of realistic data. Our tool provides new capabilities for exploring and predicting touch performance, even in the early stages of design. This is the demonstration of our accompanying paper ${ }^{1}$.
\end{abstract}

\section{Author Keywords}

Touch interaction; Modelling; Performance prediction.

\section{ACM Classification Keywords}

H.5.2 Information interfaces (e.g. HCI): User interfaces.

${ }^{1}$ Paper available at https://doi.org/10.1145/3173574.3174019 and tools available at ns.inria.fr/mjolnir/StEM/ 


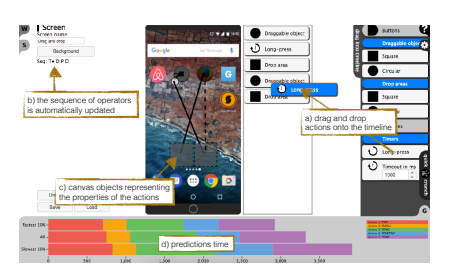

Figure 1: Example of StEM use: (a) the user drags and drops actions onto the timeline to construct the sequence of interaction of the current application screen; (b) the corresponding sequence of operators is automatically updated; (c) the user specifies the parameters of an action by manipulating its canvas representation (eg position, size, ...); (d) after querying the database, the prediction times are displayed as stacked bars (each colour represents an operator).

\section{Introduction}

Touch interfaces are now a ubiquitous means for interaction with computers. However, despite this ubiquity, it can be difficult for designers and researchers to predict how different designs for touchbased interactions will perform, and there are few tools available for exploring the performance of touch prototypes. For example, a designer may wonder whether interaction time for a common task will be reduced by adding a "bezel swipe" menu, compared to a regular menu design - and may wonder whether any performance improvement will be consistent across different screen sizes, screen orientations, and different ways of holding the device.

Several tools exist for supporting the visual design of touch interfaces, but these systems do not include performance prediction. For predictive modelling, applications such as CogTool [2] can generate predictions based on frameworks including GOMS/KLM -- but these systems do not incorporate touch actions natively, and instead approximate touch using existing mouse-based models. Recent work has provided some touch-based extensions to KLM-style models: for example, with new operators for touch actions like tapping, pointing, dragging, and flicking [4], and with time estimates for a few basic "fingerstroke-level" actions [3]. Even with these extensions, however, modelling of touch-based interactions is still limited: there is no modelling framework that provides time estimates for the full set of touch operators; in addition, the most complete existing framework was designed to predict actions in games, covers only a single device and orientation, and only provides estimates for users who are working as fast as they can [3].
As a result, it is still difficult for researchers and designers to predict user performance with touch UIs, and very difficult to do so for early design prototypes. To combine the benefits of rapid visual design tools with the capability of predictive models, we developed a new interactive tool -- the Storyboard Empirical Modelling tool (StEM) -- for exploring and predicting user performance with touch interfaces. StEM provides predictive models of six mainstream touch actions (tap point, drag, swipe, scale, and rotate), based on a large corpus of realistic data gathered from several device types, hand grips, and screen orientations. The corpus was built from a field experiment that has gathered 39,804 touch tasks from 201 users to date, and the corpus continues to grow. We characterized and compared different factors within this dataset, and found that there are significant performance differences between different devices, different screen orientations, and different hand grips, showing that designers can benefit from predictions that cover a wider range of usage situations.

The StEM tool provides new power for designers and researchers who need to understand user performance with touch interfaces. At any stage in the development of touch prototypes, StEM provides comprehensive and accurate estimates of touch performance, and it provides these estimates for a wide range of usage situations involving different types of users, devices, and postures. In addition, our corpus of empirical data and our design tool are publicly available at ns.inria.fr/ mjolnir/StEM/, and can be used by anyone who needs to more thoroughly explore touch performance. 


\section{The Storyboard Empirical Modelling tool}

We developed StEM to provide a simple and fast means for predicting user performance with touch interfaces. StEM is inspired by systems like CogTool, which take a complicated modelling framework (KLM) and provide an interactive front end that allows broad access to the underlying formalism. As described above, however, CogTool does not include several kinds of touch actions, and models others as composites of existing mousebased actions.

StEM allows designers to build touch-interaction sequences on top of visual representations of an interface - whether these are rough sketches, wireframes, screenshots of actual prototypes, or even blank screens. Instead of analysing an interaction sequence and extracting touch operators by hand, StEM allows designers to drag and drop touch actions onto the interface pictures (figure1a), which places a canvas object representing the action onto the interface, and adds the action to the touch-interaction timeline (figure 1a). To specify the specific properties of an action (eg the size and position of a tap area on the device screen), the user manipulates the canvas object on the interface picture (figure 1c).

Because many tasks require interaction with several screens, the designer can link individual screens together into a scenario. A scenario is therefore the unit at which a designer models a high-level task on a particular device - so, for a given scenario, the size of the device has to be set (ie width and height in millimetres).

Once a scenario has been designed, StEM automatically computes the corresponding sequence of operators using a set of predefined rules (described below). Each operator is associated with an index of difficulty, a time, or a movement direction depending on its type. At any time, the designer can query the database and retrieve the predicted time for the current sequence of operators. She can also specify a number of filters that specialize the predictions to specific devices, screen orientations, or hand grips. In addition, the designer can specify two percentage thresholds to obtain predictions for two user groups: the $\mathrm{x} \%$ fastest and the $\mathrm{y} \%$ slowest users in the database.

\section{Operators and rules}

The sequence of operators is computed based on previous work $[3,4]$, except that we use a different definition for tapping compared to Lee et al [3]. Lee considers tapping as a pointing task with a small amplitude (a fixed amplitude of $10 \mathrm{~mm}$ was used). In StEM, we consider tapping as a pointing action where the amplitude is unknown (ie where the starting position of the finger is unknown - as described above we use a fixed amplitude based on the screen size). Lee's tapping operator is therefore captured by our pointing operator - for example, pushing a button twice is modeled by a pointing action with an amplitude of 0 $\mathrm{mm}$ (ie an id of 0 bits).

The operators we use in StEM are: Tapping: pressing an on-screen target without knowledge of the starting finger position; Pointing: pressing an on-screen target with knowledge of the starting finger position;

Dragging: moving an on-screen object until it is within a designated area; Rotation: rotating an on-screen object with two fingers; Scaling: resizing an on-screen object with a two-finger pinch gesture; Flicking: a ballistic linear movement in one of the cardinal 
directions (up, down, left, right); Swiping: a controlled linear movement in one of the cardinal directions;

Long-press: a timeout indicating a long press on a touch target (typically 300 ms); Mental and System response: timeout representing the time taken by a user to make a decision and time out representing the time taken by the system to reach its new state. For the sake of simplicity, we gathered $M$ and $R$ into a generic operator $W$ (wait) that can be specified by the designer.

\section{Discussion}

StEM is designed to integrate support for rapid prototyping of touch interactions with the benefits of accurate performance prediction. Through drag-anddrop manipulation of touch interaction UI components, StEM users can quickly and easily specify the interaction. Having done so, performance predictions are automatically available, covering a variety of postures and form-factors, as well as enabling the designer to selectively review different performance quantiles, such as the fastest or slowest users.

A large part of the flexibility and range of the StEM tool is based on the development and validation of the data corpus (TADB) that underlies StEM's predictions. While previous modelling tools, such as CogTool, have merged prototyping and prediction capabilities, they have lacked the ability to describe many of the components of touch interaction. Although we hope that designers will choose to use StEM (once it is released), the full data corpus is available to researchers and practitioners, enabling them to repurpose the extensive dataset for their own uses.

\section{Conclusion}

Storyboard Empirical Modelling (StEM) is a drag-anddrop tool that allows designers to quickly prototype touch interactions and explore their performance implications. StEM relies on the Touch-Action database, a crowd-sourced data corpus that provides empirical characterisations of tap, point, drag, swipe, scale and rotate touch interactions across a wide range of device types, hand grips, and screen orientations. Although we developed the TADB corpus primarily as the foundation for predictions with StEM, the corpus is available on the web for other researchers and practitioners. StEM

provides new capabilities for designers and researchers who need to understand user performance with touch interfaces at any stage in the design process.

\section{References}

1. Goguey Alix, Casiez Géry, Cockburn Andy, and Gutwin Carl. 2018. Storyboard-Based Empirical Modeling of Touch Interface Performance. In Proc. of CHI. ACM.

2. Bonnie E John. 2010. Reducing the variability between novice modelers: Results of a tool for human performance modeling produced through human-centered design. In Proc. of BRIMS. Springer, 22-25.

3. Ahreum Lee, Kiburm Song, Hokyoung Blake Ryu, Jieun Kim, and Gyuhyun Kwon. 2015. Fingerstroke time estimates for touchscreen-based mobile gaming interaction. In Human Movement Science Journal. Elsevier, 211-224.

4. Andrew D. Rice and Jonathan W. Lartigue. 2014. Touch-level Model (TLM): Evolving KLM-GOMS for Touchscreen and Mobile Devices. In Proc. of ACM SE. ACM, Article 53, 6 pages. 